\title{
ON MILNOR'S INVARIANT FOR LINKS. II. THE CHEN GROUP
}

\author{
BY \\ KUNIO MURASUGI
}

1. Introduction. Let $G$ be a group and $\left\{G_{q}\right\}$ the lower central series of $G$, i.e. $G_{1}=G$ and $G_{q}=\left[G_{q-1}, G\right]$ for $q \geqq 2$. Using the second derived group $G^{\prime \prime}=\left[G^{\prime}, G^{\prime}\right]$, Chen defines a descending series of normal subgroups $\{G(q)\}$ as follows [1]: $G(1)=G$ and $G(q)=G_{q} G^{\prime \prime}$ for $q \geqq 2$. For the sake of convenience, we denote $G(\infty)=\bigcap_{q \geqq 1} G(q)$. Then the quotient group $Q(G ; q)=G(q) / G(q+1)$ is abelian. If $G$ is finitely generated, so is $Q(G ; q) . Q(G ; q)$ will be called the Chen group of $G$ in this paper.

If $G$ is the group of a (polygonal) link $L$ in the 3 -sphere, then $Q(G ; q)$ is an invariant of the link type. The objective of this paper is to show that if $L$ consists of two components then $Q(G ; q)$ is completely determined by its Alexander polynomial $\Delta(x, y)$. More precisely, a group invariant defined by means of $\Delta(x, y)$ completely determines the Chen group of the link group of $L$. If $L$ consists of more than two components, $Q(G ; q)$ may be determined by the Alexander polynomials of its subsets. In particular, if $\Delta(x, y)=0$ then $Q(G ; q)$ is free abelian and conversely. This is a characterization of a link whose Alexander polynomial vanishes. As another characterization of such a link, we shall prove that $\Delta(x, y)=0$ iff the longitudes of $L$ lie in $G(\infty)$.

We shall use the following notation.

Let $G$ be a group. $[a, b]=a b a^{-1} b^{-1}$, for $a, b \in G .\left[a_{1}, \ldots, a_{q}\right]=\left[\left[a_{1}, \ldots, a_{q-1}\right], a_{q}\right]$ for $a_{i} \in G .\left\langle a_{1}, \ldots, a_{q}\right\rangle$ denotes the normal closure of $a_{1}, \ldots, a_{q} . \partial$ denotes the free derivative in a free group ring, $d$ the usual partial derivative of a function, and 0 the trivializer. Further let

$$
D^{k}\left(t_{1}^{\alpha_{1}}, \ldots, t_{\lambda}^{a_{\lambda}}\right) f=\frac{d^{k}}{d t_{1}^{\alpha_{1}}, \ldots, d t_{\lambda}^{\alpha_{\lambda}}} f
$$

where the upper suffix $k$ frequently is omitted unless it causes confusion, and let $D^{k}(\cdots)^{0} f=\left[D^{k}(\cdots) f\right]^{0}$.

2. A group invariant. Let $G$ be a finitely presented group such that

$$
\begin{aligned}
& G \text { has a presentation } \mathscr{P} \text { of the deficiency one, } \\
& \mathscr{P}(G)=\left(x_{1}, \ldots, x_{m}: r_{1}, \ldots, r_{m-1}\right)
\end{aligned}
$$

Presented to the Society, January 24, 1969 under the title The Chen's group of a link; received by the editors March 27, 1969. 
and

the commutator quotient group $G / G^{\prime}$ is a free abelian group of rank $n \geqq 1$.

Let $F$ be a free group generated by $x_{1}, \ldots, x_{m}$. Choose a free basis $\mathfrak{A}=\left\{t_{1}, \ldots, t_{n}\right\}$ of $A_{n}=G / G^{\prime}$. Let $\Phi: F \rightarrow G$ and $\Psi: G \rightarrow A_{n}$ be natural homomorphisms( $\left.{ }^{1}\right)$. The Alexander matrix $M(\mathscr{P}, \mathfrak{A})=\left\|\partial r_{i} / \partial x_{j}\right\|^{\Phi \Psi}$ associated to $\mathscr{P}$ and $\mathfrak{A}$ is an $(m-1) \times m$ matrix over the integral group ring $Z A_{n}$. The greatest common divisor $\Delta_{\mathscr{V}}\left(t_{1}, \ldots, t_{n}\right)$ of all $(m-1) \times(m-1)$ minors of $M(\mathscr{P}, \mathfrak{A})$ will be called the Alexander polynomial of $(\mathscr{P}, \mathfrak{A})$. This is an integer polynomial on $t_{1}, \ldots, t_{n}$ with possibly negative exponents and is determined uniquely up to a unit $\pm t_{1}^{\lambda_{1}} \ldots t_{n}^{\lambda_{n}}$ in $Z A_{n}$.

Now $\Delta_{\mathfrak{V}}\left(t_{1}, \ldots, t_{n}\right)$ depends upon the choice of a basis $\mathfrak{A}$. In other words, $\Delta_{\mathfrak{U}}\left(t_{1}, \ldots, t_{n}\right)$ itself is not a group invariant. (See an example below.) However, by means of $\Delta_{\mathfrak{U}}\left(t_{1}, \ldots, t_{n}\right)$, we can define a sequence of numerical group invariants $\left\{A^{(k)}\right\}$.

Definition 2.1. Let $A^{(-1)}(\mathfrak{A})=0$ and define $A^{(0)}(\mathfrak{A})=\mathrm{ab}\left[\Delta_{\mathfrak{Y}}\left(t_{1}, \ldots, t_{n}\right)\right]^{0}\left({ }^{2}\right)$. Inductively, we suppose that $A^{(l)}(\mathfrak{U})$ is defined for $0 \leqq l \leqq k-1$. For any sequence $1 \leqq i_{1} \leqq i_{2} \leqq \cdots \leqq i_{k} \leqq n$, we set

$$
A_{i_{1}, i_{2}, \ldots, i_{k}}^{(k)}(\mathfrak{U})=\operatorname{ab}\left\{\frac{1}{\alpha_{1} ! \cdots \alpha_{n} !} D^{k}\left(t_{i_{1}}, \ldots, t_{i_{k}}\right)^{0} \Delta_{\mathscr{U}}\left(t_{1}, \ldots, t_{n}\right)\right\},
$$

where $\alpha_{j}$ is the number of occurances of $j$ in the sequence $i_{1}, \ldots i_{k}$. Let $d$ be the g.c.d. of $A^{(0)}(\mathfrak{A}), \ldots, A^{(k-1)}(\mathfrak{A})$. Let $\bar{A}_{i_{1}, \ldots, i_{k}}^{(k)}(\mathfrak{H})$ be the smallest nonnegative integer such that $\bar{A}_{\mathfrak{i}_{1}, \ldots, i_{k}}^{(k)} \equiv A_{i_{1}, \ldots, i_{k}}^{(k)} \bmod d$. Then we define

$$
A^{(k)}(\mathfrak{U})=\text { g.c.d. }\left\{\bar{A}_{i_{1}, \ldots, i_{k}}^{(k)}(\mathfrak{U})\right\}
$$

where $i_{1}, \ldots, i_{k}$ run over all permutations $1,2, \ldots, n$, subject to $i_{1} \leqq i_{2} \leqq \cdots \leqq i_{k}$. If all $\bar{A}_{i_{1}, \ldots, i_{k}}^{(k)}(\mathfrak{U})$ are zero, we define $A^{(k)}(\mathfrak{U})=0$.

From the definition, the following is evident

$$
A^{(k)}(\mathfrak{U}) \text { is a nonnegative integer and } A^{(k)}(\mathfrak{U})=0
$$

for a sufficiently large $k$.

THEOREM 2.1. $\left\{A^{(k)}(\mathfrak{A})\right\}$ is a group invariant. That is to say, $\left\{A^{(k)}(\mathfrak{H})\right\}$ is independent of the choice of presentation and free basis $\mathfrak{A}$ of $G / G^{\prime}$. Thus it may be denoted by $\left\{A^{(k)}(G)\right\}$.

Proof. First we should note that $A^{(k)}(\mathfrak{U})$ does not depend upon a particular choice of the Alexander polynomial.

(1) The same symbols will be used for the natural extensions between integral group rings.

$\left({ }^{2}\right)$ ab means "the absolute value of". 
Now, from the invariance of the elementary ideals of the Alexander matrix (Chapter VIII, (4.5) in [2]), we need only show that $\left\{A^{(k)}(\mathfrak{Q})\right\}$ is independent of the choice of basis of $G / G^{\prime}$.

Let $\mathscr{B}=\left\{s_{1}, \ldots, s_{n}\right\}$ be another basis of $G / G^{\prime}$. Then $\mathscr{B}$ is obtained from $\mathfrak{A}$ by a finite sequence of the following four transformations.

$$
\begin{array}{rlr}
\text { (i) } & t_{1} \rightarrow t_{1}^{-1}, t_{i} \rightarrow t_{i} & \text { for } i \geqq 2, \\
\text { (ii) } & t_{1} \rightarrow t_{1} t_{2}, t_{i} \rightarrow t_{i} & \text { for } i \geqq 2, \\
\text { (iii) } & t_{1} \rightarrow t_{2}, t_{2} \rightarrow t_{1}, t_{i} \rightarrow t_{i} & \text { for } i \geqq 3, \\
\text { (iv) } & t_{1} \rightarrow t_{i+1} & \text { for } 1 \leqq i \leqq n-1, t_{n} \rightarrow t_{1} .
\end{array}
$$

Therefore, it is enough to show that $A^{(k)}(\mathfrak{H})$ is unaltered under each transformation . From the definition, it follows immediately that $A^{(k)}(\mathfrak{U})$ is unaltered under (iii) or (iv).

Suppose that $\mathscr{B}$ is obtained from $\mathfrak{A}$ by (i) or (ii). Then it suffices to show the theorem for $n=2$. Let $\mathscr{B}=\left\{t_{1}^{\varepsilon} t_{2}^{\eta}, t_{2}\right\}, \varepsilon= \pm 1, \eta=0$ or 1 . By a remark given at the beginning of this proof, we may assume without loss of generality that $\Delta_{\mathscr{B}}\left(t_{1}, t_{2}\right)$ $=\Delta_{\mathfrak{U}}\left(t_{1}^{\varepsilon} t_{2}^{\eta}, t_{2}\right)$. Since $A^{(0)}(\mathscr{B})=\left|\Delta_{\mathscr{B}}(1,1)\right|=\left|\Delta_{\mathfrak{U}}(1,1)\right|=A^{(0)}(\mathfrak{Q})$, we can assume inductively that $A^{(l)}(\mathfrak{A})=A^{(l)}(\mathscr{B})$ for $l \leqq k-1$.

Let $w(\alpha, \beta)$ be a sequence $1,1, \ldots, 1,2,2, \ldots, 2$ of length $k$, where there are $\alpha$ 1's and $\beta$ 2's. Now by a substitution $t_{1}=s_{1}^{\varepsilon} s_{2}^{\eta}$ and $t_{2}=s_{2}, \Delta_{\mathfrak{A}}\left(t_{1}, t_{2}\right)$ becomes the Alexander polynomial $\Delta_{\mathscr{D}}\left(s_{1}, s_{2}\right)$ associated to $\mathscr{B}$. Therefore,

$$
A_{w(\alpha, \beta)}^{(k)}(\mathscr{B})=\mathrm{ab}\left\{\frac{1}{\alpha ! \beta !} D\left(s_{1}^{\alpha}, s_{2}^{\beta}\right)^{0} \Delta_{\mathscr{B}}\left(s_{1}, s_{2}\right)\right\}
$$

By means of the chain rule and the usual rule for differentiating product, we obtain

$$
\begin{aligned}
\frac{1}{\alpha ! \beta !} D\left(s_{1}^{\alpha}, s_{2}^{\beta}\right)^{0} \Delta_{\mathscr{S}}\left(s_{1}, s_{2}\right)= & \frac{1}{\alpha ! \beta !} \sum_{\gamma=0}^{\beta}\left(\begin{array}{l}
\beta \\
\gamma
\end{array}\right) D\left(t_{1}^{\alpha+\gamma}, t_{2}^{\beta-\gamma}\right)^{0} \Delta_{\mathscr{U}}\left(t_{1}, t_{2}\right) \varepsilon^{\alpha} \eta^{\beta} \\
& +\sum_{0 \leqq \lambda+\mu<\alpha+\beta} f_{\lambda, \mu}\left(s_{1}, s_{2}\right)^{0} D\left(t_{1}^{\lambda}, t_{2}^{\mu}\right)^{0} \Delta_{\mathfrak{V}}\left(t_{1}, t_{2}\right),
\end{aligned}
$$

where $f_{\lambda, \mu}\left(s_{1}, s_{2}\right)$ is a certain polynomial on $s_{1}$ and $s_{2}$. Since $D\left(t_{1}^{\lambda}, t_{2}^{\mu}\right)^{0} \Delta_{\mathfrak{Y}}\left(t_{1}, t_{2}\right)$ $\equiv 0 \bmod A^{(\lambda+\mu)}(\mathfrak{A})$, it follows from $(2.5)$ that

$$
A_{w(\alpha, \beta)}^{(k)}(\mathscr{B}) \equiv \mathrm{ab}\left\{\sum_{\gamma=0}^{\beta} \varepsilon^{\alpha} \eta^{\gamma} A_{w(\alpha+\gamma, \beta-\gamma)}^{(k)}(\mathfrak{H})\right\} \cdot \bmod \text { g.c.d. }\left\{A^{(0)}(\mathfrak{H}), \ldots, A^{(k-1)}(\mathfrak{H})\right\}
$$

This implies that $A^{(k)}(\mathscr{B})=A^{(k)}(\mathfrak{A})$.

COROllary. If $G / G^{\prime}$ is infinite cyclic, then $A^{(0)}(G)=1$ and $A^{(k)}(G)=0$ for $k \geqq 1$.

If $G$ is the group of a link $L, A^{(k)}(G)$ will be denoted by $A^{(k)}(L)$. 
EXAMPLE.
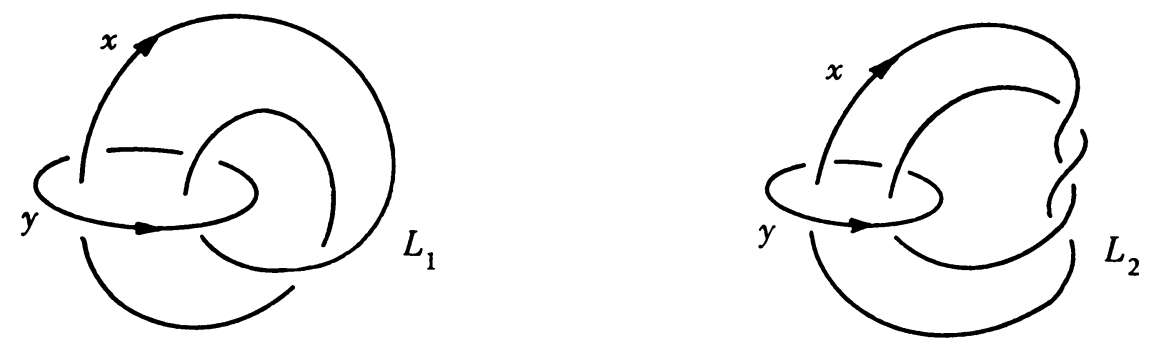

The group of two links $L_{1}$ and $L_{2}$ are isomorphic, since they have the same presentation $\left(a, b: a^{2} b=b a^{2}\right)$. However, the Alexander polynomials are distinct. In fact, $\Delta_{1}(x, y)=x y+1$ and $\Delta_{2}(x, y)=x^{3} y+1$. Then $A^{(0)}\left(L_{1}\right)=A^{(0)}\left(L_{2}\right)=2, A^{(1)}\left(L_{1}\right)$ $=A^{(1)}\left(L_{2}\right)=1$ and $A^{(k)}\left(L_{1}\right)=A^{(k)}\left(L_{2}\right)=0$ for $k \geqq 2$. Although $L_{1}$ and $L_{2}$ are of different isotopy types, their Milnor invariants are coincident: $\mu(12)=2, \mu(112)=$ $\mu(122)=1$ and $\mu\left(i_{1} i_{2} \cdots i_{n}\right)=0$ for $n \geqq 4$.

3. Structure of $F(q) / F(q+1)$. Let $F$ be a free group with $m$ free generators, $x_{1}, \ldots, x_{m}, m<\infty$. Let $\mathscr{S}(F)$ denote the set of generators of $F$, i.e. $\mathscr{S}(F)=$ $\left\{x_{1}, \ldots, x_{m}\right\}$. We define an order " $<$ " in $\mathscr{S}(F)$ in such a way that $x_{i}<x_{j}$ iff $i<j$. Now any element of $Q(F ; q)$ is a finite product of elements $\left[a_{1}, \ldots, a_{q}\right] F(q+1)$, $a_{i} \in F$. An element $\left[a_{1}, \ldots, a_{q}\right]$ of $F(q)$ is called a normal element (of length $q$ ) if all $a_{i}$ lie in $\mathscr{S}(F)$. Further, an element $\left[a_{1}, \ldots, a_{q}\right]$ is said to be standard if it is a normal element and if $a_{1}<a_{2}$ and $a_{1} \leqq a_{3} \leqq \cdots \leqq a_{q}$. Any standard element is of the form:

$$
\left[x_{i}, x_{j}, x_{i}, \ldots, x_{i}, x_{i+1}, \ldots, x_{i+1}, \ldots, x_{m}, \ldots, x_{m}\right] .
$$

For the sake of simplicity this element will be denoted by $\left[x_{i}, x_{j}, x_{i}^{\alpha_{i}}, \ldots, x_{m}^{\alpha_{m}}\right]$, $\alpha_{i}, \ldots, \alpha_{m}$ being nonnegative integers.

Now the group $Q(F ; q)$ is completely determined by the following.

THEOREM 3.1 (CHEN). $Q(F ; q)$ is a free abelian group whose basis consists of all standard representatives.

Theorem 3.1 follows easily from Lemmas 3.1-3.7 below.

LEMMA 3.1. If $a \equiv b_{1}^{m_{1}}, \ldots, b_{k}^{m_{k}} \bmod F(r)$, then for $q \geqq 1$

$$
\left[a, c_{1}, \ldots, c_{q}\right] \equiv \prod_{i=1}^{k}\left[b_{i}, c_{1}, \ldots, c_{q}\right]^{m_{i}} \bmod F(r+q)
$$

and for $q \geqq 2$,

$$
\left[c_{1}, \ldots, c_{q}, a\right] \equiv \prod_{i=1}^{k}\left[c_{1}, \ldots, c_{q}, b_{i}\right]^{m_{i}} \bmod F(r+q) .
$$


LEMMA 3.2. For $q \geqq 3,\left[a_{1}, a_{2}, a_{3}, \ldots, a_{q}\right] \equiv\left[a_{2}, a_{1}, a_{3}, \ldots, a_{q}\right]^{-1} \bmod F^{\prime \prime}$.

LEMMA 3.3. $\left[a_{1}, a_{2}, a_{3}, \ldots, a_{q}\right] \equiv\left[a_{1}, a_{2}, a_{\sigma(3)}, \ldots, a_{\sigma(q)}\right] \bmod F^{\prime \prime}$, where $\sigma$ is $a$ permutation of $3, \ldots, q$.

LEMMA 3.4.

$\left[a_{1}, a_{2}, a_{3}, a_{4}, \ldots, a_{q}\right]\left[a_{3}, a_{1}, a_{2}, a_{4}, \ldots, a_{q}\right]\left[a_{2}, a_{3}, a_{1}, a_{4}, \ldots, a_{q}\right] \equiv 1 \bmod F^{\prime \prime}$.

Since Lemmas 3.1-3.4 can easily be verified by induction, proofs will be omitted. (Cf. Lemmas A.1, A.3 and Corollary in [1].)

Let $f$ be an element of $F$. Let $S_{r}=S_{r}\left(\alpha_{1}, \ldots, \alpha_{m}\right)$ be the set of all (proper) shuffle $\left({ }^{3}\right)$ of $\alpha_{1}$ 1's, $\alpha_{2} 2$ 's, $\ldots, \alpha_{m} m$ 's, where $\alpha_{1}+\cdots+\alpha_{m}=r$. For $\omega=a_{1} a_{2} \cdots a_{r}$ $\in S_{r}\left(\alpha_{1}, \ldots, \alpha_{m}\right)$, we denote

$$
\frac{\partial^{r} f}{\partial \omega}=\frac{\partial^{r} f}{\partial x_{a_{1}} \cdots \partial x_{a_{r}}} \quad \text { and } \quad D^{r}(\omega)=D^{r}\left(x_{a_{1}}, \ldots, x_{a_{r}}\right) .
$$

Then the following lemma is easy.

LEMMA 3.5. If $f \in F^{\prime \prime}$, then for any $\omega$ of $S_{r}$ and $x_{j}$ in $\mathscr{S}(F)$,

$$
D^{r}(\omega)^{0}\left(\partial f / \partial x_{j}\right)=0 .
$$

If $f \in F_{q+1}$, then for any $\omega$ of $S_{r}\left(\partial^{r} f / \partial \omega\right)^{0}=0,0 \leqq r \leqq q$.

Further we can prove

LEMMA 3.6.

$$
\frac{1}{\alpha_{1} ! \cdots \alpha_{m} !} D\left(x_{1}^{\alpha_{1}}, \ldots, x_{m}^{\alpha_{m}}\right)^{0} f^{\phi}=\sum_{\omega}\left(\frac{\partial^{r} f}{\partial \omega}\right)^{0}\left(^{4}\right)
$$

where $\omega$ runs over all elements in $S_{r}$.

Proof. For $m=1$, the lemma follows from (3.9) in [3]. Thus we may assume inductively that (3.3) holds for $m-1$. In other words,

$$
\frac{1}{\alpha_{1} ! \cdots \alpha_{m-1} !} D\left(x_{1}^{\alpha_{1}}, \ldots, x_{m-1}^{\alpha_{m}-1}\right)^{0} f^{\phi}=\sum_{\tau}\left(\frac{\partial^{r-\alpha_{m}} f}{\partial \tau}\right),
$$

where $\tau$ runs over all elements in $S_{r-\alpha_{m}}\left(\alpha_{1}, \ldots, \alpha_{m-1}, 0\right)$. Since any element of $S_{r}\left(\alpha_{1}, \ldots, \alpha_{m}\right)$ is obtained as a generalized shuffle of a certain element of $S_{r-\alpha_{m}}$ and $\alpha_{m} m$ 's, it follows from Lemma 3.3 in [4] that

$$
\begin{aligned}
\sum_{\omega}\left(\frac{\partial^{r} f}{\partial \omega}\right)^{0} & =\left[\sum_{\tau}\left(\frac{\partial^{r-\alpha_{m}} f}{\partial \tau}\right)^{0}\right]\left[\frac{\partial^{\alpha_{m}} f}{\partial x_{m}^{\alpha_{m}}}\right]^{0} \\
& =\frac{1}{\alpha_{1} ! \cdots \alpha_{m-1} !}\left[D\left(x_{1}^{\alpha_{1}}, \ldots, x_{m-1}^{\alpha_{m}-1}\right)^{0} f^{\phi}\right] \frac{1}{\alpha_{m} !}\left[D\left(x_{m}^{\alpha_{m}}\right)^{0} f^{\phi}\right] \\
& =\frac{1}{\alpha_{1} ! \cdots \alpha_{m} !} D\left(x_{1}^{\alpha_{1}}, \ldots, x_{m}^{\alpha_{m}}\right)^{0} f^{\phi}
\end{aligned}
$$

$\left({ }^{3}\right)$ For the definition, see [4, p. 82].

$\left({ }^{4}\right) \phi$ denotes the natural homomorphism from $Z F$ onto $Z\left(F / F^{\prime}\right)$. 
where $\omega$ and $\tau$, respectively, run over all elements in $S_{r}$ and $S_{r-\alpha_{m}}$.

From Lemmas 3.5 and 3.6, we see

Lemma 3.7. If $f \in F(q+1)$, then for any $\omega \in S_{r}$ and $x_{j} \in \mathscr{S}(F)$,

$$
D^{r}(\omega)^{0}\left(\partial f / \partial x_{j}\right)^{\phi}=0, \quad 0 \leqq r<q .
$$

Lemma 3.8. Let $g$ be an element of $F(q)$. Then

$$
\left.g \equiv \prod\left[x_{i}, x_{j}, x_{i}^{\alpha_{i}}, x_{i+1}^{\alpha_{i}+1}, \ldots, x_{m}^{\alpha_{m}}\right]^{\beta\left(i, j, \alpha_{i}\right.}, \ldots, \alpha_{m}\right) \bmod F(q+1),
$$

where $1 \leqq i \leqq j \leqq m, \alpha_{i}+\cdots+\alpha_{m}=q-2$ and

$$
\beta\left(i, j, \alpha_{i}, \ldots, \alpha_{m}\right)=\frac{(-1)^{q}}{\left(\alpha_{i}+1\right) ! \alpha_{i+1} ! \cdots \alpha_{m} !} D^{q-1}\left(x_{i}^{\alpha_{i}+1}, x_{i+1}^{\alpha_{i}+1}, \ldots, x_{m}^{\alpha_{m}}\right)^{0}\left(\frac{\partial g}{\partial x_{j}}\right)^{\phi} \text {. }
$$

RemarK. This lemma may be regarded as a generalization of (5.5) in [3].

Proof. Let $g=\prod\left[x_{i}, x_{j}, x_{i}^{\alpha_{i}}, \ldots, x_{m}^{\alpha_{m}}\right]^{\beta} \cdot z$, where $z \in F(q+1)$. Since

$$
\left(\frac{\partial}{\partial x_{j}}\left[x_{i}, x_{j}, x_{i}^{\alpha_{i}}, \ldots, x_{m}^{\alpha_{m}}\right]^{\beta}\right)=(-1)\left(1-x_{i}\right)^{\alpha_{i}+1}\left(1-x_{i+1}\right)^{\alpha_{i+1}} \cdots\left(1-x_{m}\right)^{\alpha_{m}} \beta
$$

it follows that

$$
\left(\frac{\partial g}{\partial x_{j}}\right)^{\phi}=\sum(-1)\left(1-x_{i}\right)^{\alpha_{i}+1}\left(1-x_{i+1}\right)^{\alpha_{i+1}} \cdots\left(1-x_{m}\right)^{\alpha_{m}} \beta+\left(\frac{\partial z}{\partial x_{j}}\right)^{\phi} .
$$

Since $D^{q-1}(\cdots)^{0}\left(\partial z / \partial x_{j}\right)^{\phi}=0$ by Lemma 3.7, we obtain from (3.5) that

$$
\frac{1}{\left(\alpha_{i}+1\right) ! \alpha_{i+1} ! \cdots \alpha_{n} !} D\left(x_{i}^{\alpha_{i}+1}, \ldots, x_{m}^{\alpha_{m}}\right)^{0}\left(\frac{\partial g}{\partial x_{j}}\right)^{\phi}=(-1)^{a} \beta .
$$

This completes the proof.

COROLlaRY (CHEN). For $q \geqq 2$, the rank of $Q(F ; q)$ is

$$
(q-1)\left(\frac{m+q-2}{q}\right)
$$

In particular, if $m=2$, then $Q(F ; q) \cong Z^{q-1}\left({ }^{5}\right)$.

4. Subgroup $H_{\Omega}(q)$. Let $F$ be a free group and let $\Omega$ be a nonempty subset of $\mathscr{S}(F)$.

Definition 4.1. $H_{\Omega}(q)$ is the set of all elements $w$ of $F$ such that for any $s$, $0 \leqq s<q$, and for any $\alpha_{i} \in \Omega$ and $\beta \in \mathscr{S}(F)$,

$$
D\left(\alpha_{1}, \ldots, \alpha_{s}\right)^{0}(\partial w / \partial \beta)^{\phi}=0 .
$$

It is well known that $H_{\Omega}(1)=F^{\prime}$ for any $\Omega$. Further, from the definition, it follows that if $q<r$ then $H_{\Omega}(q) \supset H_{\Omega}(r)$ and if $\Omega^{\prime} \supset \Omega$ then $H_{\Omega}(q) \supset H_{\Omega^{\prime}}(q)$.

$\left.{ }^{5}\right) G^{r}$ denotes the direct product of $r$ copies of $G$. 
LEMMA 4.1. $H_{\Omega}(q)$ is a normal subgroup of $F$, and $H_{\Omega}(q) \supset F(q+1)$ for any $\Omega$.

Proof. It is obvious that $H_{\Omega}(q)$ is a subgroup of $F$. Let $g \in F$ and $u \in H_{\Omega}(q)$. Since $u \in F^{\prime}$, it follows that $u^{\phi}=1$, and hence, $\left[\partial\left(g u g^{-1}\right) / \partial \beta\right]^{\phi}=g^{\phi}(\partial u / \partial \beta)^{\phi}$. Therefore, for any $s<q, D\left(\alpha_{1}, \ldots, \alpha_{s}\right)^{0}\left[\partial\left(g_{u g}^{-1}\right) / \partial \beta\right]^{\phi}=0$. This is a proof of the first proposition. Moreover, since $H_{\Omega}(q)$ contains both $H_{\mathscr{S}}(q)$ and $F^{\prime \prime}$, and since $H_{\mathscr{S}}(q)$ contains $F_{q+1}$ by Lemma 3.5 , it follows that $H_{\Omega}(q) \supset F_{q+1} F^{\prime \prime}=F(q+1)$.

For the sake of convenience, we denote $H_{\Omega}(\infty)=\bigcap_{r \geqq 1} H_{\Omega}(r)$.

COROLlaRY. $H_{\Omega}(\infty) \supset F^{\prime \prime}$ for any $\Omega$.

Lemma 4.2. Let $g=\left[z_{1}, \ldots, z_{q}\right]$ be a normal element of $F(q)$. If $z_{i} \notin \Omega$ for some $i \geqq 3$ or if neither $z_{1}$ nor $z_{2}$ lies in $\Omega$ then $g \in H_{\Omega}(\infty)$.

Proof. For any $\beta \in \mathscr{S}(F),(\partial g / \partial \beta)^{\phi}=\left(1-z_{q}\right) \cdots\left(1-z_{3}\right)\left((\partial / \partial \beta)\left[z_{1}, z_{2}\right]\right)^{\phi}$. If $z_{i} \notin \Omega$ for $i \geqq 3$, then for any $s \geqq 0, D\left(\alpha_{1}, \ldots, \alpha_{s}\right)(\partial g / \partial \beta)^{\phi}$ contains $1-z_{i}$ as a common factor. Hence $D\left(\alpha_{1}, \ldots, \alpha_{s}\right)^{0}(\partial g / \partial \beta)^{\phi}=0$. Suppose that $z_{1}$ and $z_{2}$ are not in $\Omega$. Since

$$
\left(\frac{\partial\left[z_{1}, z_{2}\right]}{\partial \beta}\right)^{\phi}=\left(1-z_{2}\right)\left(\frac{\partial z_{1}}{\partial \beta}\right)^{\phi}+\left(z_{1}-1\right)\left(\frac{\partial z_{2}}{\partial \beta}\right)^{\phi}
$$

$D\left(\alpha_{1}, \ldots, \alpha_{s}\right)(\partial g / \partial \beta)^{\phi}$ contains either $1-z_{2}$ or $z_{1}-1$ as a common factor. Hence its trivializer vanishes.

Now we assume that there is defined a mapping $\tau$ from $\mathscr{S}(F)$ into $F$ such that

$$
g \equiv \tau(g) \quad \bmod F(2)
$$

Let $N(q)$ be the subgroup generated by all normal elements in $F(q)$. We shall extend $\tau$ to a mapping from $N(q)$ to $F(q)$.

Definition 4.2. Let $g=\left[z_{1}, \ldots, z_{q}\right]$ be a normal element of $F(q)$. If all $z_{i}$ lie in $\Omega$ then $\tau(g)=g$. Otherwise let $z_{i}$ be the first element not in $\Omega$. Then $\tau(g)=\left[z_{1}, \ldots\right.$, $\left.z_{i-1}, \tau\left(z_{i}\right), z_{i+1}, \ldots, z_{q}\right] . \tau$ is extended in the obvious way to a mapping from $N(q)$ to $F(q)$, which will be denoted by the same letter $\tau$. In particular, we define $\tau(1)=1$.

From (4.2) and Lemma 3.1, it follows

$$
\text { For any } g \in N(q), \quad g \equiv \tau(g) \quad \bmod F(q+1) \text {. }
$$

Further, since $g^{\phi}=g^{\tau \phi}$ for any $g \in N(q)$, we see that the following lemma is an easy consequence of Lemma 4.2.

LEMMA 4.3. Let $g=\left[z_{1}, \ldots, z_{q}\right]$ be a normal element of $F(q)$. If $z_{i}$ is not in $\Omega$ for $i \geqq 3$ or if neither $z_{1}$ nor $z_{2}$ lies in $\Omega$, then $\tau(g) \in H_{\Omega}(\infty)$.

By means of $\tau$, we shall define a sequence of mappings $\left\{\tau_{n}\right\}$ from $F$ to $F(n)$.

First, we extend the order in $\mathscr{S}(F)$ lexicographically to an order in the set of normal elements of the same length. More precisely, we say $\left[a_{1}, \ldots, a_{q}\right]<$ $\left[b_{1}, \ldots, b_{q}\right]$ iff $a_{1}<b_{1}$ or $a_{1}=b_{1}, \ldots, a_{m}=b_{m}, a_{m+1}<b_{m+1}, 0<m<q$.

Using this order, we can write any element of $F(q) / F(q+1)$ as a finite product of standard elements in a unique way (Theorem 3.1). Namely, for any $g \in F(q)$, 
$g \equiv \prod_{i=1}^{k} g_{i}^{\alpha_{i}} \bmod F(q+1)$, where $g_{1}<g_{2}<\cdots<g_{k}$ and $\alpha_{i}$ is a nonzero integer. This rule $\rho_{q}$ which to an element $g$ of $F(q)$ associates a finite product of standard elements $\prod_{i=1}^{k} g_{i}^{\alpha_{i}}$ will be called the standardization. If $g$ is a normal element of $F(q)$, then $g \equiv \rho_{q}(g) \bmod F^{\prime \prime}$.

Now we define $\tau_{n}$ inductively as follows:

For any $g \in F, \tau_{1}(g)=g \in F(1)=F$. Suppose that $\tau_{n}(g)$ is defined for $n \geqq 1$. Then we define $\tau_{n+1}(g)=\tau_{n}(g)\left[\tau \rho_{n} \tau_{n}(g)\right]^{-1}$. Since $\tau_{n}(g) \equiv \tau \rho_{n} \tau_{n}(g) \bmod F(n+1)$, it follows that $\tau_{n+1}(g) \in F(n+1)$.

LEMMA 4.4. If $g \in F(n)$, then $\tau_{r}(g)=g$ for $r \leqq n$.

Proof. For $n=1$, Lemma 4.4 is trivial. Assume that Lemma 4.4 holds for $n-1$. Since $g \in F(n)$ and $\tau_{n-1}(g)=g \equiv 1 \bmod F(n)$, it follows that $\tau_{n}(g)=\tau_{n-1}(g) \tau(1)^{-1}$ $=\tau_{n-1}(g)=g$.

Lemma 4.5. If $g \in F(n) \cap H_{\Omega}(\infty)$ then $\tau_{n+1}(g) \in F(n+1) \cap H_{\Omega}(\infty)$.

Proof. Let $\rho_{n}(g)=\prod_{i} g_{i}^{\alpha_{i}}$. Since $F(n) \ni g, \tau_{n}(g)=g$, and hence, $\tau_{n+1}(g)$ $=g\left(\tau \rho_{n}(g)\right)^{-1}$. Since $\tau_{n+1}(g) \in F(n+1)$, we only need to show that $\tau_{n+1}(g)$ $\in H_{\Omega}(\infty)$, or equivalently, $\tau \rho_{n}(g) \in H_{\Omega}(\infty)$. Now, since $g \in H_{\Omega}(\infty)$, it follows from Lemma 3.8 that $g_{i}$ cannot be of the form: $\left[z_{1}, z_{2}, z_{3}, \ldots, z_{n}\right]$, where all $z_{i}$ except possibly $z_{2}$ are in $\Omega$. Thus, Lemma 4.4 follows from Lemma 4.3.

5. Main Lemma. Let $F$ be a free group with two disjoint nonempty sets of generators $\Omega=\left\{x_{1}, \ldots, x_{n}\right\}$ and $\Gamma=\left\{a_{1}, \ldots, a_{m}\right\}$. Let $\tau$ be a mapping from $\mathscr{S}(F)$ to $F$ defined as follows:

$$
\begin{array}{ll}
\tau\left(x_{i}\right)=x_{i}, & 1 \leqq i \leqq n, \quad \text { and } \\
\tau\left(a_{i}\right)=f_{a_{i}}^{-1} a_{i}, & 1 \leqq i \leqq m,
\end{array}
$$

where $f_{a_{i}} \in F(2)$.

We define an order in $\mathscr{S}(F)$ as follows. Elements in $\Omega$ (or $\Gamma$ ) are naturally ordered according to their indices, and any element of $\Omega$ is less than any of $\Gamma$. This order can be extended lexicographically to an order in the set of normal elements of the same length. See $\S 4$.

Since $\tau$ satisfies (4.2), a sequence of mappings $\left\{\tau_{n}\right\}$ is well defined.

Now, using $f_{a_{i}}$, we shall define three endomorphisms $\nu, \sigma$ and $\phi_{r}(r \geqq 1)$ of $F$ as follows:

$$
\begin{aligned}
& \nu\left(x_{i}\right)=x_{i} \quad \text { and } \quad \nu\left(a_{i}\right)=f_{a_{i}}, \\
& \sigma\left(x_{i}\right)=x_{i} \text { and } \sigma\left(a_{i}\right)=1 \text {, } \\
& \phi_{1}=\sigma \quad \text { and } \quad \phi_{r}=\sigma \nu^{r-1} \text {, for } r \geqq 2 .
\end{aligned}
$$

Consider an element $w=\left[W, x_{1}\right]$ in $F(2)$. We can write $w=\bar{w} \rho_{2}(w)$, where $\bar{w} \in$ $F(3)$ and $\rho_{2}(w) \in F(2)$. Since $\tau_{h}(w)=\tau_{h-1}(w)\left[\tau \rho_{h-1} \tau_{h-1}(w)\right]^{-1}$ and $\tau_{2}(w)=w$, it follows by induction that

$$
\tau_{h}(w)=w\left[\tau \rho_{2} \tau_{2}(w)\right]^{-1}\left[\tau \rho_{3} \tau_{3}(w)\right]^{-1} \cdots\left[\tau \rho_{h-1} \tau_{h-1}(w)\right]^{-1} .
$$


We should note that $\tau_{h}(w)$ is a finite product of the $\tau$-image of standard elements of the length $<h$. For convenience, we write $\tau_{h}(w)$ in the following form:

$$
\tau_{h}(w)=\bar{w} \prod_{2 \leqq k \leqq h-1} \prod_{i} g_{k, i}^{\beta(k, i)},
$$

where $g_{k, i}$ is a $\tau$-image of a standard element of $F(k)$. Therefore, $g_{k, i}^{\phi}$ is a standard element of $F(k)$.

On the other hand, since $\tau_{h}(w) \in F(h)$, we see that $\rho_{h} \tau_{h}(w)$ is written as

$$
\prod_{j} g_{h, j}^{\beta(h, j)}
$$

where $g_{h, j}$ is a standard element of $F(h)$.

The purpose of this section is to determine the exponent $\beta(k, i)$ of $g_{k, i}$ of some particular type. Denote by $\beta\left(z_{1}, \ldots, z_{r}\right)$ the exponent of the element $\left[z_{1}, \ldots, z_{r}\right]$ occurring in $\tau_{h}(w)$ or $\rho_{h} \tau_{h}(w)$. Then we shall prove

LEMMA 5.1.

$$
\beta\left(x_{i}, x_{j}, x_{i}^{\alpha_{i}}, \ldots, x_{n}^{\alpha_{n}}\right)=\frac{(-1)^{n-1}}{\alpha_{i} ! \cdots \alpha_{m} !} D\left(x_{i}^{\alpha_{i}}, \ldots, x_{n}^{\alpha_{n}}\right)^{0}\left(\frac{\partial W^{\tilde{\Phi}}}{\partial x_{j}}\right)^{\phi},
$$

where $\tilde{\phi}=\phi_{h-1}$ and $\sum_{i} \alpha_{i}=h-2$.

Now to avoid unnecessary complications, we shall prove Lemma 5.1 for $n=2$, and use $x, y$ instead of $x_{1}, x_{2}$. In fact, this is what we really need in the subsequent sections.

First we shall establish a relation between $\bar{w}$ and $W$.

LEMMA 5.2. If $z \in \mathscr{S}(F)$ is different from $x$, then for $\lambda+\mu>0$,

$$
\frac{1}{\lambda ! \mu !} D\left(x^{\lambda}, y^{\mu}\right)^{0}\left(\frac{\partial W}{\partial z}\right)^{\phi}=\frac{(-1)}{(\lambda+1) ! \mu !} D\left(x^{\lambda+1}, y^{\mu}\right)\left(\frac{\partial \bar{w}}{\partial z}\right)^{\phi} \text {. }
$$

Proof. Since $w=\bar{w} \rho_{2}(w)$,

$$
\left(\frac{\partial w}{\partial z}\right)^{\phi}=\left(\frac{\partial \rho_{2}(w)}{\partial z}\right)^{\phi}+\left(\frac{\partial \bar{w}}{\partial z}\right)^{\phi}
$$

On the other hand, $(\partial w / \partial z)^{\phi}=(1-x)(\partial W / \partial z)^{\phi}$, because of $w=[W, x]$. Therefore,

$$
(1-x)\left(\frac{\partial W}{\partial z}\right)^{\phi}=\left(\frac{\partial \rho_{2}(w)}{\partial z}\right)^{\phi}+\left(\frac{\partial \bar{w}}{\partial z}\right)^{\phi}
$$

Since $\left(\partial \rho_{2}(w) / \partial z\right)^{\phi}$ is a linear polynomial on $x, y$ or $a_{j}, D\left(x^{\lambda+1}, y^{\mu}\right)^{0}\left(\partial \rho_{2}(w) / \partial z\right)^{\phi}=0$, for $\lambda+\mu \geqq 1$. Thus from (5.5) we obtain

$$
\frac{1}{(\lambda+1) ! \mu !}\left(\begin{array}{c}
\lambda+1 \\
1
\end{array}\right)(-1) D\left(x^{\lambda}, y^{\mu}\right)^{0}\left(\frac{\partial W}{\partial z}\right)^{\phi}=\frac{1}{(\lambda+1) ! \mu !} D\left(x^{\lambda+1}, y^{\mu}\right)^{0}\left(\frac{\partial \bar{w}}{\partial z}\right)^{\phi} .
$$

This is the required formula. 
The next lemma is a recursion formula involving $\beta\left(z_{1}, \ldots, z_{r}\right)$. Let $u_{i}=\tau\left(a_{i}\right)$.

LEMMA 5.3. For $p+q \leqq h-2, p \geqq 1, p+q \geqq 2$,

$$
\begin{aligned}
\beta\left(x, u_{i}, x^{p-1}, y^{q}\right)= & \sum_{r, s, k} \frac{1}{p ! q !} r ! s !(-1)^{r+s+p+q-1} \beta\left(x, u_{k}, x^{r-1}, y^{s}\right)\left(\begin{array}{l}
p \\
r
\end{array}\right)\left(\begin{array}{l}
q \\
s
\end{array}\right) \\
& \times D\left(x^{p-r}, y^{q-s}\right)^{0}\left(\frac{\partial u_{k}}{\partial a_{i}}\right)^{\phi}+\frac{(-1)^{p+q}}{(p-1) ! q !} D\left(x^{p-1}, y^{q}\right)^{0}\left(\frac{\partial W}{\partial a_{i}}\right)^{\phi},
\end{aligned}
$$

where the summation runs over all $r, s$ and $k$ such that $1 \leqq r \leqq p, 0 \leqq s \leqq q, 1 \leqq r+s$ $\leqq p+q-1,1 \leqq k \leqq m$.

Proof. To obtain the required formula, we shall calculate $D\left(x^{p}, y^{q}\right)^{0}\left(\partial \tau h / \partial a_{i}\right)^{\phi}$ from (5.3). Since $p+q \geqq 2, D\left(x^{p}, y^{q}\right)^{0}\left(\left(\partial / \partial a_{i}\right)\left[z_{1}, z_{2}\right]\right)^{\phi}=0$ for $z_{i} \in \mathscr{S}(F)$. Also, since $g_{k, j}$ is a $\tau$-image of a standard element of $F(k)$, it follows from Lemma 4.3 that $g_{k, j}=\left[z_{1}, \ldots, z_{k}\right] \in H_{\Omega}(\infty)$ if $z_{i}=u_{l}$ for some $i \geqq 3$ or both $z_{1}^{\phi}$ and $z_{2}^{\phi}$ are in $\Gamma$. Therefore, by excluding those factors which are contained in $H_{\Omega}(\infty)$, we obtain:

For $p+q \geqq 2$,

$$
\begin{aligned}
D\left(x^{p}, y^{q}\right)^{0}\left(\frac{\partial \tau_{h}(w)}{\partial a_{i}}\right)^{\phi}=D\left(x^{p}, y^{q}\right)^{0}\left\{\sum_{r, s, k, 1 \leqq r+s \leqq p+q}(-1) \beta\left(x, u_{k}, x^{r-1}, y^{s}\right)\right. \\
\left.\times(1-x)^{r}(1-y)^{s}\left(\frac{\partial u_{k}}{\partial a_{i}}\right)^{\phi}+\left(\frac{\partial \bar{w}}{\partial a_{i}}\right)^{\phi}\right\} .
\end{aligned}
$$

Since $\left(\partial u_{k} / \partial a_{i}\right)^{\phi^{0}}=\delta_{i, k}$ and $D\left(x^{p}, y^{q}\right)^{0}\left(\partial \tau_{h}(w) / \partial a_{i}\right)=0$ for $p+q \leqq h-2$, because $\tau_{h}(w) \in F(h)$, we see that if $p+q \geqq 2$,

$$
\begin{aligned}
0= & \sum \sum \beta\left(x, u_{k}, x^{r-1}, y^{s}\right)\left(\begin{array}{l}
p \\
r
\end{array}\right)\left(\begin{array}{l}
q \\
s
\end{array}\right) r ! s !(-1)^{r+s-1} D\left(x^{p-r}, y^{q-s}\right)^{0}\left(\frac{\partial u_{k}}{\partial a_{i}}\right)^{\phi} \\
& +(-1)^{p+q-1} p ! q ! \beta\left(x, u_{i}, x^{p-1}, y^{q}\right)+D\left(x^{p}, y^{q}\right)^{0}\left(\frac{\partial \bar{w}}{\partial a_{i}}\right) .
\end{aligned}
$$

From this formula and Lemma 5.2, Lemma 5.3 follows.

LEMMA 5.4. For any $q, 2 \leqq q \leqq h-2$,

$$
\begin{aligned}
\beta\left(y, u_{i}, y^{q-1}\right)= & \sum_{1 \leqq s \leqq q-1} \sum_{k} \frac{1}{q !} s !(-1)^{s+q-1} \beta\left(y, u_{k}, y^{s-1}\right)\left(\begin{array}{l}
q \\
s
\end{array}\right) \\
& \times D\left(y^{q-s}\right)^{0}\left(\frac{\partial u_{k}}{\partial a_{i}}\right)^{\phi}+\frac{(-1)^{q}}{q !} D\left(y^{q}\right)^{0}\left(\frac{\partial \bar{w}}{\partial a_{i}}\right)^{\phi} .
\end{aligned}
$$

Proof. From (5.3), it follows that if $q \geqq 2$,

$$
\begin{aligned}
0= & D\left(y^{q}\right)^{0}\left(\frac{\partial \tau_{h}(w)}{\partial a_{i}}\right) \\
= & D\left(y^{q}\right)^{0}\left\{(-1) \beta\left(y, a_{i}\right)(1-y)+\sum_{k} \sum_{1 \leqq s \leqq q} \beta\left(y, u_{k}, y^{s-1}\right)(1-y)^{s}(-1)\left(\frac{\partial u_{k}}{\partial a_{i}}\right)^{\phi}+\left(\frac{\partial \bar{w}}{\partial a_{i}}\right)^{\phi}\right\} \\
= & \sum_{k} \sum_{1 \leqq s<q}(-1)^{s-1} \beta\left(y, u_{k}, y^{s-1}\right) s !\left(\begin{array}{l}
q \\
s
\end{array}\right) D\left(y^{q-s}\right)^{0}\left(\frac{\partial u_{k}}{\partial a_{i}}\right)^{\phi} \\
& +(-1)^{q-1} q ! \beta\left(y, u_{k}, y^{q-1}\right)+D\left(y^{q}\right)^{0}\left(\frac{\partial \bar{w}}{\partial a_{i}}\right)^{\phi} .
\end{aligned}
$$


As a special case of Lemmas 5.3 and 5.4, we have

LEMMA 5.5. $\beta\left(x, u_{i}\right)=\beta\left(x, a_{i}\right)=(-1)\left(\partial W / \partial a_{i}\right)^{\phi 0}$.

Proof. From the expression

$$
w=[W, x]=\bar{w}[x, y]^{\beta(x, y)} \prod_{i}\left[x, a_{i}\right]^{\beta\left(x, a_{i}\right)},
$$

it follows that

$$
\left(\frac{\partial w}{\partial a_{i}}\right)^{\phi}=(1-x)\left(\frac{\partial W}{\partial a_{i}}\right)^{\phi}=\left(\frac{\partial \bar{w}}{\partial a_{i}}\right)^{\phi}+(x-1) \beta\left(x, a_{i}\right)
$$

Thus, $D(x)^{0}\left(\partial w / \partial a_{i}\right)^{\phi}=(-1)\left(\partial W / \partial a_{i}\right)^{\phi 0}$. On the other hand, $D(x)^{0}\left(\partial \bar{w} / \partial a_{i}\right)^{\phi}=0$. Hence we have $D(x)^{0}\left(\partial W / \partial a_{i}\right)^{\phi}=\beta\left(x, a_{i}\right)$. Thus, Lemma 5.5 follows.

Now we are in position to prove Lemma 5.1.

Since Lemma 5.1 is trivial for $h=2$, we assume that $h>2$. Since $\tau_{h}(w) \equiv$ $\rho_{h} \tau_{h}(w) \bmod F(h+1)$, it follows from Lemma 3.7 that

$$
D\left(x^{\lambda+1}, y^{\mu}\right)^{0}\left(\frac{\partial \tau_{h}(w)}{\partial y}\right)^{\phi}=D\left(x^{\lambda+1}, y^{\mu}\right)^{0}\left(\frac{\partial \rho_{h} \tau_{h}(w)}{\partial y}\right)^{\phi} \text { for } \lambda+\mu+2=h .
$$

Thus, Lemma 3.8 shows that

$$
\beta\left(x, y, x^{\lambda}, y^{\mu}\right)=\frac{(-1)^{\lambda+\mu}}{(\lambda+1) ! \mu !} D\left(x^{\lambda+1}, y^{\mu}\right)^{0}\left(\frac{\partial \tau_{h}(w)}{\partial y}\right)^{\phi},
$$

where $h=\lambda+\mu+2$. By a direct calculation, the right-hand side of (5.8) becomes

$$
\begin{array}{r}
\frac{(-1)^{\lambda+\mu}}{(\lambda+1) ! \mu !} D\left(x^{\lambda+1}, y^{\mu}\right)^{0}\left\{\sum_{i} \sum_{p, q}(-1) \beta\left(x, u_{i}, x^{p-1}, y^{q}\right)\right. \\
\left.\cdot(1-x)^{p}(1-y)^{q}\left(\frac{\partial u_{i}}{\partial y}\right)^{\phi}+\left(\frac{\partial \bar{w}}{\partial y}\right)^{\phi}\right\} \\
=\frac{(-1)^{\lambda+\mu}}{(\lambda+1) ! \mu !}\left\{\sum \sum p ! q !(-1)^{p+q+1}\left(\begin{array}{c}
\lambda+1 \\
p
\end{array}\right)\left(\begin{array}{c}
\mu \\
q
\end{array}\right) \beta\left(x, u_{i}, x^{p-1}, y^{q}\right)\right. \\
\left.\cdot D\left(x^{\lambda+1-p}, y^{\mu-q}\right)^{0}\left(\frac{\partial u_{i}}{\partial y}\right)^{\phi}+D\left(x^{\lambda+1}, y^{\mu}\right)^{0}\left(\frac{\partial \bar{w}}{\partial y}\right)^{\phi}\right\},
\end{array}
$$

where the summation runs over all $p, q$ and $i$ such that $1 \leqq p \leqq \lambda+1,0 \leqq q \leqq \mu$, $1 \leqq p+q \leqq \lambda+\mu, 1 \leqq i \leqq m$.

Now consider the right-hand side of (5.4). By the chain rule, we have

$$
\begin{aligned}
(-1)^{\lambda+\mu-1} \frac{1}{\lambda ! \mu !} & D\left(x^{\lambda}, y^{\mu}\right)^{0}\left(\frac{\partial W^{\phi_{h-1}}}{\partial y}\right)^{\phi} \\
& =\frac{(-1)^{\lambda+\mu-1}}{\lambda ! \mu !} D\left(x^{\lambda}, y^{\mu}\right)^{0}\left\{\left(\frac{\partial W}{\partial y}\right)^{\phi}+\sum_{i}\left(\frac{\partial W}{\partial a_{i}}\right)^{\phi}\left(\frac{\partial a_{i}^{\phi_{h-1}}}{\partial y}\right)^{\phi}\right\}
\end{aligned}
$$

Then by Lemma 5.2, the first term of the right-hand side of (5.10) coincides with 
the last term of the right-hand side of (5.9). Thus we only need to show that

$$
\begin{array}{r}
\frac{1}{(\lambda+1) ! \mu !} \sum \sum\left(\begin{array}{c}
\lambda+1 \\
p
\end{array}\right)\left(\begin{array}{l}
\mu \\
q
\end{array}\right) p ! q !(-1)^{p+q} \beta\left(x, u_{i}, x^{p-1}, y^{q}\right) \\
\cdot D\left(x^{\lambda+1-p}, y^{\mu-q}\right)^{0}\left(\frac{\partial u_{i}}{\partial y}\right)^{\phi} \\
=\frac{1}{\lambda ! \mu !} D\left(x^{\lambda}, y^{\mu}\right)^{0} \sum_{i}\left(\frac{\partial W}{\partial a_{i}}\right)^{\phi}\left(\frac{\partial a_{i}^{\phi}-1}{\partial y}\right)^{\phi}
\end{array}
$$

where $p, q$ run over the same range as defined in (5.8).

Now the chain rule shows that

$$
\frac{\partial a_{i}^{\phi_{h-1}}}{\partial y}=\frac{\partial a_{i}^{v}}{\partial y}+\sum_{k} \frac{\partial a_{i}^{v}}{\partial a_{k}} \frac{\partial a_{k}^{\phi_{h-2}}}{\partial y} \text { and } \frac{\partial a_{i}^{\phi_{2}}}{\partial y}=\frac{\partial u_{i}}{\partial y}
$$

Therefore, by induction, we can prove that

$$
\frac{\partial a_{i}^{\phi_{h-1}}}{\partial y}=\sum_{i \leqq j, \ldots, k, l \leqq m}\left(\frac{\partial a_{i}^{v}}{\partial a_{j}}\right) \quad \ldots \quad\left(\frac{\partial a_{k}^{v}}{\partial a_{l}}\right)\left(\frac{\partial u_{l}}{\partial y}\right) .
$$

Noting that $\left(\partial a_{i}^{v} / \partial a_{j}\right)^{0}=0$, we see that

$$
\begin{aligned}
& D\left(x^{r}, y^{s}\right)^{0}\left(\frac{\partial a_{i}^{\phi_{h-1}}}{\partial y}\right)^{\phi} \\
& \quad=\left(\begin{array}{l}
r \\
b
\end{array}\right)\left(\begin{array}{l}
s \\
c
\end{array}\right)\left[D\left(x^{b}, y^{c}\right)^{0} \sum\left(\frac{\partial a_{i}^{v}}{\partial a_{j}}\right) \cdots\left(\frac{\partial a_{k}^{v}}{\partial a_{l}}\right)\right] D\left(x^{r-b}, y^{s-c}\right)^{0}\left(\frac{\partial u_{l}}{\partial y}\right)^{\phi} .
\end{aligned}
$$

By substituting (5.7) and (5.13) into (5.11), we obtain an expression involving $\beta\left(x, u_{i}, x^{r-1}, y^{s}\right)$ and $D\left(x^{r}, y^{s}\right)\left(\partial u_{l} / \partial y\right)^{\phi}$. Compare the term involving $D\left(x^{\lambda+1-p}, y^{\mu-q}\right)$ $\cdot\left(\partial u_{i} / \partial y\right)^{\phi}$. In the left-hand side of $(5.11)$, the coefficient of this term is

$$
\frac{1}{(\lambda+1) ! \mu !}(-1)^{p+q}\left(\begin{array}{c}
\lambda+1 \\
p
\end{array}\right)\left(\begin{array}{c}
\mu \\
q
\end{array}\right) \beta\left(x, u_{i}, x^{p-1}, y^{q}\right)
$$

On the other hand, in the right-hand side of (5.11), it is

$$
\frac{1}{\lambda ! \mu !}\left(\begin{array}{c}
\lambda \\
p-1
\end{array}\right)\left(\begin{array}{l}
\mu \\
q
\end{array}\right) D\left(x^{p-1}, y^{q}\right)^{0}\left[\sum\left(\frac{\partial W}{\partial a_{j}}\right) \cdots\left(\frac{\partial a_{k}^{v}}{\partial a_{i}}\right)\right]^{\phi} .
$$

Thus it is enough to show that these expressions coincide. Then, by a direct computation, it reduces to

$$
(-1)^{p+q} \beta\left(x, u_{i}, x^{p-1}, y^{q}\right)=\frac{1}{(p-1) ! q !} D\left(x^{p-1}, y^{q}\right)^{0}\left[\left(\frac{\partial W}{\partial a_{j}}\right) \cdots\left(\frac{\partial a_{k}^{v}}{\partial a_{i}}\right)\right]^{\phi}
$$

Now (5.16) will be proved by induction on $p+q$.

In the case where $p+q=1$, i.e. $p=1$ and $q=0$, (5.16) becomes $(-1) \beta\left(x, u_{i}\right)$ $=\left(\partial W / \partial a_{i}\right)^{0}$. This is true by Lemma 5.5 . 
Suppose (5.16) holds for $r+s<p+q$. Then

$$
\begin{aligned}
&(-1)^{p+q} \beta\left(x, u_{i}, x^{p-1}, y^{q}\right) \\
&=\sum \sum \frac{1}{p ! q !} r ! s !(-1)^{r+s-1}\left(\begin{array}{l}
p \\
r
\end{array}\right)\left(\begin{array}{l}
q \\
s
\end{array}\right) \frac{(-1)^{r+s-1}}{(r-1) ! s !} D\left(x^{r-1}, y^{s}\right)^{0} \\
& \cdot\left[\sum\left(\frac{\partial W}{\partial a_{j}}\right) \cdots\left(\frac{\partial a_{k}^{v}}{\partial a_{l}}\right)\left(\frac{\partial u_{l}}{\partial a_{t}}\right)\right]^{\phi} D\left(x^{p-r}, y^{q-s}\right)^{0}\left(\frac{\partial u_{t}}{\partial a_{i}}\right)^{\phi} \\
&+\frac{1}{(p-1) ! q !} D\left(x^{p-1}, y^{q}\right)^{0}\left(\frac{\partial W}{\partial a_{i}}\right)^{\phi} \\
&= \frac{1}{(p-1) ! q !} D\left(x^{p-1}, y^{q}\right)^{0}\left(\frac{\partial W}{\partial a_{i}}\right)^{\phi}+\sum \frac{1}{p ! q !} r ! s !\left(\begin{array}{l}
p \\
r
\end{array}\right)\left(\begin{array}{l}
q \\
s
\end{array}\right) \frac{1}{(r-1) ! s !} D\left(x^{r-1}, y^{s}\right)^{0} \\
& \cdot\left[\left(\frac{\partial W}{\partial a_{j}}\right) \cdots\left(\frac{\partial a_{k}^{v}}{\partial a_{l}}\right)\left(\frac{\partial u_{l}}{\partial a_{t}}\right)\right]^{\phi} D\left(x^{p-r}, y^{q-s}\right)^{0}\left(\frac{\partial u_{t}}{\partial a_{i}}\right)^{\phi} \cdot
\end{aligned}
$$

Since

$$
\frac{1}{p !} \frac{1}{q !} r ! s !\left(\begin{array}{l}
p \\
r
\end{array}\right)\left(\begin{array}{l}
q \\
s
\end{array}\right) \frac{1}{(r-1) ! s !}=\frac{1}{(p-1) ! q !}\left(\begin{array}{l}
p-1 \\
r-1
\end{array}\right)\left(\begin{array}{l}
q \\
s
\end{array}\right)
$$

(5.17) becomes

$$
\begin{aligned}
(-1)^{p+q}\left(x, u_{i}, x^{p-1}, y^{q}\right) & =\frac{1}{(p-1) ! q !} D\left(x^{p-1}, y^{q}\right)^{0}\left\{\left(\frac{\partial W}{\partial a_{i}}\right)+\sum\left(\frac{\partial W}{\partial a_{k}}\right) \cdots\left(\frac{\partial u_{t}}{\partial a_{i}}\right)\right\}^{\phi} \\
& =\frac{1}{(p-1) ! q !} D\left(x^{p-1}, y^{q}\right)^{0} \sum\left\{\left(\frac{\partial W}{\partial a_{k}}\right) \cdots\left(\frac{\partial u_{t}}{\partial a_{i}}\right)\right\}^{\phi}
\end{aligned}
$$

This proves Lemma 5.1.

6. The group of a link. Let $\mathscr{P}(G)=\left(x_{i, j} ; r_{i, j}\right)$ be a Wirtinger presentation of $G$ of a link with $n$ components. The standard presentation $\mathscr{P}_{s}$ of $G$ is a presentation [6]:

$$
\mathscr{P}_{s}(G)=\left(x_{i, j}: s_{i, j}, 1 \leqq i \leqq n, 1 \leqq j \leqq \lambda_{i}\right),
$$

where $s_{i, j}=v_{i, j} x_{i, 1} v_{i, j}^{-1} x_{i, j+1}^{-1}$.

Let $\widetilde{F}=\left(x_{i, j}, 1 \leqq i \leqq n, 1 \leqq j \leqq \lambda_{i}:\right)$ and $F=\left(x_{i}, a_{i, j}, 1 \leqq i \leqq n, 2 \leqq j \leqq \lambda_{i}:\right)$. Then there exists an isomorphism $\chi: \tilde{F} \rightarrow F$ defined by

$$
\chi\left(x_{i, 1}\right)=x_{i} \text { and } \chi\left(x_{i, j}\right)=a_{i, j}^{-1} x_{i}, \quad j \geqq 2 .
$$

By means of $\chi$, we can obtain a new presentation $\mathscr{P}_{0}$ of $G$.

$$
\mathscr{P}_{0}(G)=\left(x_{i}, a_{i, j}: t_{i, j-1}, t_{i}, 1 \leqq i \leqq n, 2 \leqq j \leqq \lambda_{i}\right),
$$

where $t_{i, j-1}=\chi\left(s_{i, j-1}\right)$ and $t_{i}=\chi\left(s_{i, \lambda_{i}}\right)$.

It is evident that $t_{i, j-1}=\left[x_{i}, u_{i, j}\right]^{-1} a_{i, j}$ and $t_{i}=\left[\xi_{i}, x_{i}\right]$, where $u_{i, j}=\chi\left(v_{i, j}\right)$ and $\xi_{i}=\chi\left(v_{i, \lambda_{i}}\right)$.

In order to apply lemmas obtained in the previous sections on the link group, we shall define a mapping $\tau$. 
Let $\Omega=\left\{x_{1}, \ldots, x_{n}\right\}$ and $\Gamma=\left\{a_{i, j}\right\}$.

First, we define the order " $<$ " in $\mathscr{S}(F)=\Omega \cup \Gamma$ as follows:

$$
\begin{aligned}
& x_{i}<a_{j, k} \text { for any } i, j, k \text {, } \\
& x_{i}<x_{j} \quad \text { iff } i<j \text {, and } \\
& a_{j, k}<a_{l, m} \text { iff } j<l \text { or } j=l, k<m \text {. }
\end{aligned}
$$

We extend this order lexicographically to an order in the set of normal elements of the same length. (See $\$ \S 4$ and 5.)

Now, $\tau$ will be defined as

$$
\tau\left(a_{i, j}\right)=t_{i, j} \text { and } \tau\left(x_{i}\right)=x_{i} .
$$

Since $\tau$ satisfies (4.2) and (5.1), $\left\{\tau_{n}\right\}$ also are defined. Further, $\nu, \sigma$ and $\phi_{r}$ are defined by (5.2). With these endomorphisms and $\chi$, we introduce another endomorphism $\tilde{\nu}$ of $\tilde{F}$ and $\tilde{\sigma}$ as follows:

$$
\tilde{\nu}=\chi^{-1} \nu \chi \quad \text { and } \quad \tilde{\sigma}=\sigma \chi .
$$

Then the homomorphism $\theta_{p}$ in [6] is exactly $\tilde{\sigma} \tilde{\nu}^{p-1}, p \geqq 1$ and

$$
\theta_{p}=\phi_{p} \chi, \text { for } p \geqq 1 \text {. }
$$

Now, Theorem 3.1 shows that a free generator of $Q(F ; q)$ is represented by a standard element of $F(q)$.

Definition 6.1. A standard element $\left[z_{1}, \ldots, z_{q}\right]$ of $F(q)$ is said to be substantial if every $z_{\mathfrak{i}}$ lies in $\Omega$. Otherwise, it is insubstantial.

Now the longitude $l_{i}$ of each component $L_{i}$ of a link $L$ represents an element of the link group $G$ of $L$. By a suitable choice of the base point of $G$, we may assume without loss of generality that $l_{i}$ is represented by $t_{i}=\left[\xi_{i}, x_{i}\right]$ in $\mathscr{P}_{0}(G)$.

Suppose that $L$ has only two components. Then we can prove

THEOREM 6.1 .

$$
\frac{1}{p ! q !} D\left(x_{1}^{p}, x_{2}^{q}\right)^{0}\left(\frac{\partial \xi_{2}^{\tilde{\Phi}}}{\partial x_{1}}\right)^{\phi} \equiv \frac{(-1)^{q}}{p ! q !} D\left(x_{1}^{p}, x_{2}^{q}\right)^{0} \Delta\left(x_{1}, x_{2}\right) \bmod A^{(p+q-1)}(L)
$$

where $\tilde{\phi}=\phi_{p+q+1}$.

REMARK. (6.6) remains true if $\left(\partial \xi_{2}^{\Phi} / \partial x_{1}\right)$ is replaced by $\partial \xi_{1}^{\Phi} / \partial x_{2}$ in the left-hand side and $(-1)^{q}$ by $(-1)^{p}$ in the right-hand side.

Proof. Let $\eta_{2}$ be the element representing a longitude of the second component $L_{2}$ of $L$ in the standard presentation $\mathscr{P}_{s}(G)$.

Then (6.4) implies that $\eta_{2}^{\theta}=\xi_{2}^{\tilde{\Phi}}$, where $\theta=\theta_{p+q+1}$ and $\delta=\phi_{p+q+1}$. Thus, in order to prove (6.6) it sufficer $u$ show that

$$
\frac{1}{p ! q !} D\left(x_{1}^{p}, x_{2}^{q}\right)^{0}\left(\frac{\partial \eta_{2}^{\theta}}{\partial x_{1}}\right)^{\phi} \equiv(-1)^{q} \frac{1}{p ! q !} D\left(x_{1}^{p}, x_{2}^{q}\right)^{0} \Delta\left(x_{1}, x_{2}\right) \bmod A^{(p+q+1)}(L) .
$$


However, this is essentially what we have proved in [6]. To ensure it we shall follow the proof of Theorem 4.1 in [6]. To avoid a confusion on notation involved, it should be noted that $x_{1}, x_{2}$ in (6.7) are denoted by $x, y$ in [6]. First, for the case $p+q=0$, Theorem 6.1 is true. Now we may assume inductively that the theorem is true for $(r, s)<(p, q)$. From the chain rule, it follows that

$$
\frac{\partial \eta^{\theta}}{\partial x}=\sum\left(\frac{\partial \eta}{\partial x_{i}}\right)^{\theta}\left(\frac{\partial x_{i}^{\theta}}{\partial x}\right)+\sum\left(\frac{\partial \eta}{\partial y_{k}}\right)^{\theta} \frac{\partial y_{k}^{\theta}}{\partial x}
$$

Thus we see that

$$
\frac{1}{p ! q !} D\left(x^{p}, y^{q}\right)^{0}\left(\frac{\partial \eta^{\theta}}{\partial x}\right)^{\phi}
$$

is equal to the right-hand side of (6.1) in [6]. On the other hand, (7.1) in [6] holds. By the induction assumption, we obtain

$$
\frac{1}{r !} \frac{1}{s !} D\left(x^{r}, y^{s}\right)^{0} \Delta(x, y) \equiv 0 \bmod A^{(p+q-1)}(L) .
$$

Thus the same argument as was used in $\$ \$ 7-8$ in [6] shows that we need only prove that

$$
\begin{aligned}
X_{i}^{\theta}(r, s)^{0} & \equiv \Gamma_{i}^{p, q}(p-r, q-s) \bmod A^{(p+q-1)} \\
Y_{k}^{\theta}(r, s)^{0} & \equiv \Lambda_{k}^{p, q}(p-r, q-s) \bmod A^{(p+q-1)}
\end{aligned}
$$

If in $\S 8$ in [6] we replace modulo $\Delta^{*}([p+1, q+1])$ by $\bmod A^{(p+q-1)}$, the proof given there goes through. Thus (6.6) holds.

7. A presentation of the Chen group of $L$. In this section we shall find a presentation of $Q(G ; q)$ for the link group $G$.

Lemma 7.1. Let $\mathscr{P}_{0}(G)=\left(x_{i}, a_{i, j}: t_{i, j}, t_{i}\right)$ be a presentation of the link group $G$ given in $\S 6$. Let $R=\left\langle t_{i, j}, t_{i}\right\rangle$. Suppose that $F(q) \cap R=\left\langle w_{1}, \ldots, w_{r}, \ldots, w_{p}\right\rangle, w_{i} \in F$, where $w_{1}, \ldots, w_{r}$ are linearly independent $\bmod F(q+1)$ and $w_{r+1}, \ldots, w_{p} \in F(q+1)$. Then $F(q+1) \cap R=\left\langle\left[w_{i}, x_{j}\right],\left[w_{i}, a_{k, l}\right], w_{r+1}, \ldots, w_{p}, 1 \leqq i \leqq r, 1 \leqq j, k \leqq n, 2 \leqq l \leqq \lambda_{k}\right\rangle$.

Proof. In Lemma A5 [1] put $G=F, H=F(q+1), M=F(q)$ and $N=F(q) \cap R$. Then $H \subset M$ and $[M, G]=[F(q), F] \subset F(q+1)$. Then the lemma follows immediately from Lemma A5 in [1].

Considei the following disjoint set $W_{i, j}$ of $F(q)$.

$$
\begin{aligned}
& W_{1,1}=\left\{t_{i, j}\right\}, \\
& W_{1, q}=\left\{\left[x_{k}, t_{i, j}, z_{1}, \ldots, z_{q-2}\right]\right\} \text { for } q \geqq 2, \\
& W_{2, q}=\left\{\left[t_{i, j}, a_{k, l}, z_{1}, \ldots, z_{q-2}\right]\right\} \text { for } q \geqq 2, \text { and } \\
& W_{3, q}=\left\{\left[x_{i}, x_{j}, z_{1}, \ldots, z_{k-1}, t_{l, m}, z_{k+1}, \ldots, z_{q-2}\right]\right\} \quad \text { for } q \geqq 3,
\end{aligned}
$$

where $z_{i} \in \mathscr{S}(F)$ and the $\phi$-image of any elements in $W_{i, j}$ is standard. Let $W_{q}=$ $\bigcup_{i=1}^{3} W_{i, q}$. 
We should note that if $g$ is an insubstantial generator of $F(q)$ then $\tau(g)$ is contained in $W_{q}$.

LemMA 7.2. The set $W_{q}$ is linearly independent $\bmod F(q+1)$.

Proof. Since $t_{i j} \equiv a_{i j} \bmod F(2), W_{q}$ is in one-one correspondence to the set of insubstantial generators of $F(q)$. Thus the lemma follows.

Lemma 4.3 implies

$$
W_{2, q} \cup W_{3, q} \subset H_{\Omega}(\infty) .
$$

In the following, we always assume that $n=2$, i.e. $L$ has only two components and we use $x, y$ instead of $x_{1}, x_{2} ; \zeta$ instead of $\xi_{2}$.

Now consider $t_{2}=[\zeta, y]$. Let $p$ be the first integer such that $\tau_{p}\left(t_{2}\right)^{\sigma} \not \equiv 1 \bmod F(p+1)$. Such an integer $p$ may not exist. If it exists, it is uniquely determined. Thus $p$ will be denoted by $p(G)$. For the sake of convenience, we say $p(G)=\infty$ if $p$ does not exist.

Lemma 7.3. Let $A^{(k)}(L)$ be the first nonzero member of $\left\{A^{(k)}(L)\right\}$. Then $p(G)=k+1$. Consequently, $p(G)=\infty$ iff $\Delta(x, y)=0$.

A proof follows immediately from Lemma 5.1 and Theorem 6.1.

Now we define a new set $R_{q}$ in $F(q)$ as follows.

For $q<p(G), R_{q}=\left\{\tau_{q+1}\left(t_{2}\right)\right\}$ and for $q \geqq p(G)=p$,

$$
R_{q}=\left\{\left[\tau_{p}\left(t_{2}\right), x^{\lambda}, y^{\mu}\right], 0 \leqq \lambda, \mu \leqq q-p, \lambda+\mu=q-p\right\} .
$$

Lemma 7.4. For $q \geqq p(G), R_{q} \cup W_{q}$ is linearly independent $\bmod F(q+1)$.

Proof. Suppose $\tau_{p}\left(t_{2}\right)^{\sigma} \equiv \prod\left[x, y, x^{\gamma}, y^{\delta}\right]^{\beta\left(x, y, x^{\gamma}, y^{\delta}\right)} \bmod F(p+1)$. Since $\tau_{p}\left(t_{2}\right)^{\sigma} \not \equiv$ $1 \bmod F(p+1)$, some $\beta$ are not zero. Then

$$
\left.\left[\tau_{p}\left(t_{2}\right), x^{\lambda}, y^{\mu}\right] \equiv \prod_{\gamma, \delta}\left[x, y, x^{\lambda+\gamma}, y^{\mu+\delta}\right]^{\beta\left(x, y, x^{\gamma}, y^{\delta}\right.}\right) \bmod F(q+1)\left\langle W_{q}\right\rangle .
$$

This implies the lemma.

LEMMA 7.5. Let $g=[W, z]$ be an element of $F(q), q \geqq 3$, where $W \in W_{q-1}$ and $z \in \mathscr{S}(F)$. Let $\bar{g}=g^{\phi \rho \tau}$. Then $r(g)=g \bar{g}^{-1}$ is in $H_{\Omega}(\infty) \cap F(q+1)$. $\rho$ denotes the standardization $\rho_{q}$.

Proof. Suppose $W \in W_{1, q-1}$, i.e. $W=\left[x_{k}, t_{i, j}, z_{1}, \ldots, z_{q-3}\right]$. Then $g=\left[x_{k}, t_{i, j}, z_{1}\right.$, $\left.\ldots, z_{q-3}, z\right]$. If $z \geqq x_{k}$, then from Lemma 3.3 we see that $g$ is congruent to an element

$$
g_{1}=\left[x_{k}, t_{i, j}, z_{1}, \ldots, z_{p}, z, z_{p+1}, \ldots, z_{q-3}\right] \bmod F^{\prime \prime},
$$

where $g_{1}^{\phi}$ is standard. Since $g^{\phi \rho}=g_{1}^{\phi}, \bar{g}=g^{\phi \rho \tau}=g_{1}^{\phi \tau}=g_{1}$. Thus $r(g)=g \bar{g}^{-1}=g g_{1}^{-1} \in F^{\prime \prime}$ $\subset H_{\Omega}(\infty) \cap F(q+1)$.

If $z<x_{k}$, then from Lemmas 3.3 and 3.4, it follows easily that $g \equiv g_{1}^{-1} g_{2} \bmod F^{\prime \prime}$, where $g_{1}=\left[z, x_{k}, z_{1}, \ldots, z_{p}, t_{i, j}, z_{p+1}, \ldots, z_{q-3}\right]$ and $g_{2}=\left[z, t_{i, j}, x_{k}, z_{1}, \ldots, z_{q-3}\right]$. 
We should note that $\boldsymbol{g}_{1}^{\phi}, \boldsymbol{g}_{2}^{\phi}$ are standard and $\boldsymbol{g}_{1}^{\phi}<\boldsymbol{g}_{2}^{\phi}$. Let $z_{m}$ be the first element in $\Gamma$ occuring in a sequence $z_{1}, \ldots, z_{p}, a_{i, j}$. Then, since $\tau\left(z_{m}\right)=t_{r, s} \equiv z_{m} \bmod F(2)$, it follows from 3.1 that

$g_{1} \equiv g_{1}^{\prime}=\left[z_{1}, x_{k}, z_{1}, \ldots, z_{m-1}, t_{r, s}, z_{m+1}, \ldots, z_{p}, a_{i, j}, z_{p+1}, \ldots, z_{q-3}\right] \bmod F(q+1)$ and hence, $g \equiv g_{1}^{\prime-1} g_{2} \bmod F(q+1)$. Since $g^{\phi \rho}=\left(g_{1}^{\prime \phi}\right)^{-1} g_{2}^{\phi}, \bar{g}=g^{\phi \rho \tau}=\left(\left(g_{1}^{\prime \phi}\right)^{-1} g_{2}^{\phi}\right)^{\tau}$ $=g_{1}^{\prime-1} g_{2}$. Therefore, $r(g)=g \bar{g}^{-1}=g\left(g_{1}^{\prime-1} g_{2}\right)^{-1} \in F(q+1)$. Next, we have to show that $r(g) \in H_{\Omega}(\infty)$. Since $g_{1}^{\prime}$ satisfies the assumption in Lemma 4.2, $g_{1}^{\prime} \in H_{\Omega}(\infty)$. Therefore, Lemma 4.3 shows that $g_{2}^{\prime \phi \tau}=g_{1}^{\prime} \in H_{\Omega}(\infty)$. Thus, it remains only to show that $g_{2}$ and $g$ belong to $H_{\Omega}(\infty)$.

We consider two cases.

Case 1. Some element in $\Gamma$ occurs in a sequence $z_{1}, \ldots, z_{q-3}$.

Then, from Lemma 4.2 we see that $g^{\phi}$ and $g_{2}^{\phi}$ belong to $H_{\Omega}(\infty)$, and hence, both $g=g^{\phi \tau}$ and $g_{2}=g_{2}^{\phi \tau}$ are in $H_{\Omega}(\infty)$.

Case 2. $z_{i} \in \Omega$ for all $i$.

Then $g_{2}=\left[z, t_{i, j}, x_{k}, z_{1}, \ldots, z_{q-3}\right]$ and $g_{1}=\left[z, x_{k}, z_{1}, \ldots, z_{q-3}, t_{i, j}\right]$. Since $\left(g_{1}^{\phi}\right)^{-1} g_{2}^{\phi}=g^{\phi \rho}, \bar{g}=g^{\phi \rho \tau}=\left(g_{1}^{\phi \tau}\right)^{-1} g_{2}^{\phi \tau}=g_{1}^{-1} g_{2}$. Therefore, $r(g)=g \bar{g}^{-1}=g\left(g_{1}^{-1} g_{2}\right)^{-1}$ $\in F^{\prime \prime} \subset F(q+1) \cap H_{\Omega}(\infty)$.

Thus, we have proved that if $W \in W_{1, q-1}$ then $r(g) \in H_{\Omega}(\infty) \cap F(q+1)$.

In the other case where $W \in W_{2, q-1}$ or $W_{3, q-1}$, the exact same method is available, but the proof is much shorter, because we already know that $g$ and $\bar{g}$ are in $H_{\Omega}(\infty)$. So we shall omit the details.

LEMMA 7.6. $R \cap F(q)=\left\langle W_{q}, R_{q}, K_{q}\right\rangle$, where $K_{q}$ is a certain collection of elements in $H_{\Omega}(\infty) \cap F(q+1)$ and it will be defined in the proof.

Proof. In the case $q=1$, since $F(1) \cap R=R=\left\langle t_{i, j}, t_{i}\right\rangle$, Lemma 7.6 is certainly true, where $K_{1}$ is empty. Suppose that Lemma 7.6 is true for $r<q$. First we assume that $q<p(G)$. Since $R_{q}$ and $K_{q}$ are subsets of $F(q+1)$, Lemma 7.1 implies that $F(q+1) \cap R=\left\langle\tilde{W}_{q+1}, K_{q}, R_{q}\right\rangle$, where $\tilde{W}_{q+1}=\left\{[w, z], w \in W_{q}, z \in \mathscr{S}(F)\right\}$. We note that $\tilde{W}_{q+1} \supset W_{q+1}$. Take an element $g=[w, z]$ from $\tilde{W}_{q+1}-W_{q+1}$. Then by Lemma 7.5, $r(g)=g\left(g^{\phi \rho \tau}\right)^{-1}$ is in $H_{\Omega}(\infty) \cap F(q+1)$. Let $K_{q+1}^{\prime}$ be the totality of $r(g)$ for $g \in \tilde{W}_{q+1}-W_{q+1}$. This will be a part of $K_{q+1}$ sought. Since, for any standard generator $f$ of $F(q+1), f^{\imath}$ is in $W_{q+1}$, we see that $\left\langle\tilde{W}_{q+1}\right\rangle=\left\langle W_{q+1}, K_{q+1}^{\prime}\right\rangle$. Next, take an element $g$ from $K_{q}$. Then Lemma 4.5 shows that $\tau_{q+2}(g) \in H_{\Omega}(\infty) \cap$ $F(q+2)$. Let $K_{q+1}^{\prime \prime}$ denote the totality of $\tau_{q+2}(g)$ for $g \in K_{q}$. Then it is verified that $\left\langle W_{q+1}, K_{q}\right\rangle=\left\langle W_{q+1}, K_{q+1}^{\prime \prime}\right\rangle$. Let $K_{q+1}=K_{q+1}^{\prime} \cup K_{q+1}^{\prime \prime}$. Then $\left\langle\tilde{W}_{q+1}, K_{q}\right\rangle=\left\langle W_{q+1}\right.$, $\left.K_{q+1}^{\prime}, K_{q}\right\rangle=\left\langle W_{q+1}, K_{q+1}\right\rangle$. Similarly, we can prove that $\left\langle R_{q}, W_{q+1}\right\rangle=\left\langle R_{q+1}\right.$, $\left.W_{q+1}\right\rangle$. Thus $R \cap F(q+1)=\left\langle W_{q+1}, R_{q+1}, K_{q+1}\right\rangle$.

Now consider the case where $q \geqq p(G)$. Since $W_{q} \cup R_{q}$ is linearly independent $\bmod F(q+1)$, it follows that $F(q+1) \cap R=\left\langle\tilde{W}_{q+1}, K_{q}, \tilde{R}_{q+1}\right\rangle$, where $\tilde{R}_{q+1}$ $=\left\{\left[\tau_{p}\left(t_{2}\right), x^{\lambda}, y^{\mu}, z\right]\right\}$. Of course, $\widetilde{R}_{q+1} \supset R_{q+1}$. Let $K_{q+1}^{\prime}, K_{q+1}^{\prime \prime}$ be the same sets as are defined in the previous paragraphs. Then $\left\langle\tilde{W}_{q+1}, K_{q}\right\rangle=\left\langle W_{q+1}, K_{q+1}^{\prime}, K_{q+1}^{\prime \prime}\right\rangle$. 
We shall define the third set $K_{q+1}^{\prime \prime \prime}$. Take an element $g$ from $\tilde{R}_{q+1}-R_{q+1} . g$ is of the form: $\left[\tau_{p}\left(t_{2}\right), x^{\lambda}, y^{\mu}, z\right]$. If $z$ is in $\Gamma$ then $g \in H_{\Omega}(\infty) \cap F(q+1)$. Thus $s(g)=\tau_{q+2}(g)$ is in $H_{\Omega}(\infty) \cap F(q+2)$ by Lemma 4.5. If $z$ is in $\Omega$ then $z=x$ and $s(g)=$ $g\left[\tau_{p}\left(t_{2}\right), x^{\lambda+1}, y^{\mu}\right]^{-1}$ lies in $F^{\prime \prime}$ and hence, $s(g) \in H_{\Omega}(\infty) \cap F(q+2)$. Let $K_{q+1}^{\prime \prime \prime}$ be the totality of $s(g)$ for $g \in \widetilde{R}_{q+1}-R_{q+1}$. Then $\left\langle R_{q+1}\right\rangle=\left\langle R_{q+1}, K_{q+1}^{\prime \prime \prime}\right\rangle$. Let $K_{q+1}=$ $K_{q+1}^{\prime} \cup K_{q}^{\prime \prime} \cup K_{q+1}^{\prime \prime \prime}$. Then $\left\langle\tilde{W}_{q+1}, \widetilde{R}_{q+1}, K_{q}\right\rangle=\left\langle W_{q+1}, R_{q+1}, K_{q+1}\right\rangle$. This proves the lemma.

LEMMA 7.7. If $g$ is an insubstantial generator of $F(q)$, then $g F(q+1)$ is contained in $(F(q) \cap R) F(q+1)$.

Proof. We know from Lemma 7.6 that $F(q) \cap R=\left\langle W_{q}, R_{q}, K_{q}\right\rangle$. Let $g=$ $\left[z_{1}, \ldots, z_{q}\right]$ and $z_{i}$ the first element of $\Gamma$ occurring in the sequence $z_{1}, \ldots, z_{q}$. Then, since $z_{i} \equiv t_{j, k} \bmod F(2)$, we see that

$$
\begin{aligned}
g F(q+1) & =\left[z_{1}, \ldots, z_{q}\right] F(q+1) \\
& =\left[z_{1}, \ldots, z_{i-1}, t_{j, k}, z_{i+1}, \ldots, z_{q}\right] F(q+1) \\
& \subset W_{q} F(q+1) \subset(F(q) \cap R) F(q+1),
\end{aligned}
$$

From Lemmas 7.6 and 7.7 , we obtain immediately

LEMMA 7.8. For the link group $G$,

$$
Q(G ; q)=\left(\left[x, y, x^{\lambda}, y^{\mu}\right], 0 \leqq \lambda, \mu \leqq q-2, \lambda+\mu=q-2: R_{q}^{\sigma}, F(q+1)^{\sigma}\right) .
$$

Let $\tau_{p(G)}\left(t_{2}\right)^{\sigma} \equiv \prod\left[x, y, x^{\lambda}, y^{\mu}\right]^{\beta(\lambda, \mu)} \bmod F(p+1)$. Since not all $\beta(\lambda, \mu)$ are zero, $d=$ g.c.d. ${ }_{\lambda, \mu}\{\beta(\lambda, \mu)\}$ is not zero. Then from Lemma 7.8 , it follows

LEMMA 7.9.

$$
\begin{aligned}
& Q(G ; q) \cong Z^{q-1} \quad \text { for } q<p(G)=p, q \geqq 2, \\
& Q(G ; p) \cong Z_{d}+Z^{p-2} .
\end{aligned}
$$

8. Determination of $Q(G ; q)$. To determine $Q(G ; q)$ for $q \geqq p(G)+1$, we need the following

LEMMA 8.1. Let

$$
M(m, n)=\left[\begin{array}{ccccccc}
a_{1} & \cdots & a_{n} & & & 0 \\
& a_{1} & \cdots & a_{n} & & \\
& & \ddots & \ddots & \ddots & \\
0 & & & a_{1} & \cdots & a_{n}
\end{array}\right]
$$

be an $m \times(n+m-1)$ matrix with entries in the integer ring $Z$. Let $\varepsilon_{\lambda}(M)$ be the ideal generated by $\lambda \times \lambda$ minors of $M(m, n)$. Then $\varepsilon_{\lambda}(M)=\left(d^{\lambda}\right)$, where $d=$ g.c.d. $\left\{a_{1}, \ldots, a_{n}\right\}$ and $1 \leqq \lambda \leqq m$.

Proof. For $n=1$ or $m=1$, the lemma is obvious. Thus we assume that the lemma is true for $M(\bar{m}, \bar{n}),(\bar{m}, \bar{n})<(m, n)$. Consider $\varepsilon_{\lambda}(M(m, n))$. First we should 
note that it is sufficient to show that $\varepsilon_{\lambda}(M)=Z$ if $d=1$. Since $\varepsilon_{\lambda}(M(m, n)) \supset$ $\varepsilon_{\lambda}(M(m-1, n))=Z$, it follows that for $1 \leqq \lambda<m, \varepsilon_{\lambda}(M(m, n))=Z$ and hence, it remains to show that $\varepsilon_{m}(M(m, n))=Z$. Or equivalently, $m \times m$ minors of $M(m, n)$ generate $Z$.

Now by the induction assumption, we know that for the matrix

$$
\begin{aligned}
& N(m-1, n-1)=\left[\begin{array}{ccccccc}
0 & a_{2} & \cdots & a_{n} & & 0 \\
& 0 & a_{2} & \cdots & a_{n} & & \\
& 0 & \ddots & \ddots & \ddots & \ddots & \\
& & & 0 & a_{2} & \cdots & a_{n}
\end{array}\right], \\
& \varepsilon_{\lambda}(N)=\left(d_{1}^{\lambda}\right), \quad 1 \leqq \lambda<m, \cdot d_{1}=\text { g.c.d. }\left(a_{2}, \ldots, a_{n}\right) .
\end{aligned}
$$

Consider the set $\tilde{M}(j)$ of all $m \times m$ minors of $M(m, n)$ that contains $(1, j)$ entry $a_{j}$. $\tilde{M}(j)$ generates an ideal $K_{j}$ in $Z$. Then $\varepsilon_{m}(M)=K_{1}+\cdots+K_{n}$.

We may assume that $a_{1} \neq 0$, otherwise by the induction assumption, we are done. Then $K_{1}=\left(a_{1}\right)$, since $\varepsilon_{m-1}(M(m-1, n))=Z$. Consider the matrix $\tilde{N}=$ $N(m-1, n-1)$. Then by the induction assumption, $\varepsilon_{m-1}(\tilde{N})=\left(d_{1}^{m-1}\right)$. Therefore, $K_{2} \subset \varepsilon_{m-1}(\tilde{N})+\left(a_{1}\right)=\left(d_{1}^{m-1}\right)+\left(a_{1}\right)$. On the other hand, any element $\tilde{D}$ of $\varepsilon_{m-1}(\tilde{N})$ can be written as $\tilde{D}=a_{2} D+a_{1} b$ for some $m \times m$ minor $D$ of $\tilde{M}(2)$ and some integer $b$. Thus $\varepsilon_{m-1}(\tilde{N}) \subset K_{2}+K_{1}$. Therefore, $K_{1}+K_{2} \subset K_{1}+\varepsilon_{m-1}(\tilde{N}) \subset K_{1}+K_{2}$.

Since $d_{1}$ and $a_{1}$ are relatively prime, so are $d_{1}^{m-1}$ and $a_{1}$. Thus $\varepsilon_{m}(M) \supset K_{1}$ $+K_{2}=Z$.

From Lemmas 7.9 and 8.1, it follows that

LEMMA 8.2. $Q(G ; q) \cong Z_{d}^{q-p}+Z^{p-1}$ for $q \geqq p(G)+1$, where $d$ is the same integer as in Lemma 7.9.

On the other hand, the integer $d$ can be described in terms of $A^{(k)}(L)$. In fact, Lemma 5.1 and Theorem 6.1 imply that

$$
\begin{aligned}
A^{(q)}(L) & =0 \quad \text { for } q<p-1, \\
A^{(p-1)}(L) & =d .
\end{aligned}
$$

Thus we have finally:

THEOREM 8.1. Let $L$ be a link with two components. Let $d=A^{(p-1)}(L)$ be the first nonzero member of $\left\{A^{(k)}(L)\right\}$. Then

$$
\begin{array}{ll}
Q(G ; q) \cong Z^{q-1} & \text { for } q<p, \\
Q(G ; q) \cong Z_{d}^{q-p}+Z^{p-2} & \text { for } q \geqq p .
\end{array}
$$

COROllary. $\Delta(x, y)=0$ iff $Q(G ; q) \cong Z^{q-1}$ for any $q \geqq 2$.

As an application, we shall prove the following

THEOREM 8.2. Let $L$ be a link with two components. Then $\Delta(x, y)=0$ iff each longitude lies in $G(\infty)$. 
Proof. Let $\xi$ and $\eta$ be longitudes of $L$. From the remark given in Theorem 6.1, we see that we need only show that $\xi$ lies in $G(\infty)$ iff $\Delta(x, y)=0$. Further, Theorem 6.1 shows that we need only show that

$$
\xi \in G(\infty) \text { iff } D\left(x^{\lambda}, y^{\mu}\right)^{0}\left(\partial \xi^{\phi_{k+1}} / \partial y\right)^{\phi}=0, \text { for any } k, 0 \leqq k=\lambda+\mu .
$$

Since (8.3) is equivalent to

(8.4) $\xi \in G(n)$ iff $D\left(x^{\lambda}, y^{\mu}\right)^{0}\left(\partial \xi^{\phi_{k+1}} / \partial y\right)^{\phi}=0, \quad$ for any $k, 0 \leqq k=\lambda+\mu \leqq n-2$. we shall prove (8.4).

Now (8.4) is certainly true for $n=2$. In fact, let $\xi \equiv y^{\alpha} \prod_{i, j} a_{i, j}^{\beta_{i, j}} \bmod F(2)$. Then $\xi^{\phi_{1}} \equiv y^{\alpha} \bmod F(2)$. Therefore $\left(\partial \xi^{\phi_{1}} / \partial y\right)^{0}=\alpha=0$ implies $\xi \equiv \prod_{i, j} a_{i, j}^{\beta_{i, j}} \bmod F(2)$. Since $a_{i, j}=\left[x_{i}, u_{i j}\right]$ in $G$, we see that $a_{i j} \equiv 1 \bmod G(2)$. Thus $\xi \in G(2)$. Conversely, if $\xi \in G(2)$, then we can write

$$
\xi \equiv[x, y]^{\alpha} \prod\left[x, a_{i j}\right]^{\beta_{i j}} \prod\left[y, a_{i j}\right]^{\gamma_{i j}} \prod\left[a_{i j}, a_{k l}\right]^{\delta} \bmod F(3) .
$$

Then $\xi^{\phi_{1}} \equiv[x, y]^{\alpha} \bmod F(3)$. Thus, $\left(\partial \xi^{\phi_{1}} / \partial y\right)^{0}=0$.

Now we assume inductively that (8.4) is true for any $m<n$. Suppose that

$$
D\left(x^{r}, y^{s}\right)^{0}\left(\partial \xi^{\phi_{k+1}} / \partial y\right)^{\phi}=0
$$

for any $k, 0 \leqq k=r+s \leqq n-2$. Then by the induction assumption $\xi \in G(n-1)$. Thus we can write

$$
\xi \equiv \prod_{\lambda+\mu=n-3}\left[x, y, x^{\lambda}, y^{\mu}\right]^{\alpha(\lambda, \mu)} \prod\left[z_{1}, \ldots, z_{n-1}\right]^{\beta} \bmod F(n),
$$

where $\left[z_{1}, \ldots, z_{n-1}\right]$ is a standard element in which at least one of $z_{i}$ is in $\Gamma$. Then $\left[z_{1}, \ldots, z_{n-1}\right]^{\phi_{n-1}} \in F(n)$. Thus $\xi^{\phi_{n-1}} \equiv \prod\left[x, y, x^{\lambda}, y^{\mu}\right]^{\alpha(\lambda, \mu)} \bmod F(n)$. Then from Lemma 3.8 and our assumption, we see that

$$
D\left(x^{\lambda+1}, y^{\mu}\right)^{0}\left(\partial \xi^{\phi_{n-1}} / \partial y\right)^{\phi}=(\lambda+1) ! \mu ! \alpha(\lambda, \mu)=0 .
$$

Thus $\xi \equiv \prod\left[z_{1}, \ldots, z_{n-1}\right]^{\beta} \bmod F(n)$. Since one of $z_{i}$ is in $\Gamma$, it follows that $\left[z_{1}, \ldots, z_{n-1}\right] \equiv 1 \bmod G(n)$. Hence, $\xi \in G(n)$.

Conversely, we assume that $\xi \in G(n)$. Then we can write

$$
\xi=\prod_{\lambda+\mu=n-2}\left[x, y, x^{\lambda}, y^{\mu}\right]^{\beta(\lambda, \mu)} \prod\left[z_{1}, \ldots, z_{n}\right] \bmod F(n+1)
$$

where $\left[z_{1}, \ldots, z_{n}\right]$ is a standard element in which at least one of $z_{i}$ is in $\Gamma$. Then

$$
\xi^{\phi_{n-1}} \equiv \prod_{\lambda, \mu}\left[x, y, x^{\lambda}, y^{\mu}\right]^{\beta(\lambda, \mu)} \bmod F(n+1),
$$

since $\left[z_{1}, \ldots, z_{n}\right]^{\phi_{n-1}} \in F(n+1)$ for $n \geqq 2$. Thus for $p+q=n-2, D\left(x^{p}, y^{q}\right)^{0}\left(\partial \xi^{\phi_{n-1}} / \partial y\right)$ $=0$. This completes the proof.

\section{REFERENCES}

1. K. T. Chen, Integration in free groups, Ann. of Math. (2) 54 (1951), 147-162. MR 13, 105. 
2. R. H. Crowell and R. H. Fox, Introduction to knot theory, Ginn. Boston, Mass., 1963. MR 26 \#4348.

3. R. H. Fox, Free differential calculus. I: Derivation in the free group ring, Ann. of Math. (2) 57 (1953), 547-560. MR 14, 843.

4. R. H. Fox, K. T. Chen and C. Lyndon, Free differential calculus. IV, Ann. of Math. (2) 68 (1958), 81-95.

5. J. Milnor, Isotopy of links. Algebraic geometry and topology, A Symposium in Honor of S. Lefschetz, Princeton Univ. Press, Princeton, N. J., 1957, pp. 280-306. MR 19, 1070.

6. K. Murasugi, On Milnor's invariant for links, Trans. Amer. Math. Soc. 124 (1966), 94-110. MR 33 \#6611.

7. N. Smyth, "Boundary links," in Topology seminar, Wisconsin, 1965, R. H. Bing and R. J. Bean (editors), Ann. of Math. Studies, no. 60, Princeton Univ. Press, Princeton, N. J., 1966, pp. 69-72. MR 34 \#1974.

\section{UNIVERSITY OF TORONTO,}

Toronto, Canada 